\title{
Schock - eine Übersicht für die klinische Praxis
}

Dorothea Hempel, Guido Michels

\author{
Aufgrund der hohen Mortalität bei „Schock“ ist zügiges und adäquates Handeln \\ essenziell. Grundlage ist das Wissen über die notwendige Diagnostik, die unterschiedli- \\ chen Ätiologien und die therapeutischen Ansätze der verschiedenen Schockformen. Die- \\ ser Beitrag zeichnet gezielt den Ablauf in der Versorgung des Schockpatienten nach, vom \\ Erstkontakt bis zum Beginn der spezifischen Therapie, und bietet somit einen Leitfaden \\ für die klinische Praxis.
}

\begin{abstract}
Merke
Die Mortalität bei septischem Schock beträgt 40 - 60\% und bei kardiogenem Schock ca. $40 \%$.

In Präklinik und Klinik stellt das Management des Schocks auch im Zeitalter der Hightech-Medizin immer noch eine Herausforderung für das gesamte Behandlungsteam dar. Ein zirkulatorischer Schock ist definiert als ein Missverhältnis zwischen Sauerstoffbedarf und Sauerstoffangebot [1]. Klinisch geht ein Schock mit typischen Zeichen der Minderperfusion einher, die zur Diagnosefindung beitragen (Infobox 1). Nicht selten liegt bei der Akutvorstellung ein undifferenzierter Kreislaufschock vor mit zunächst unklarer Ätiologie - diese muss aber zur gezielten Therapie möglichst rasch geklärt werden.
\end{abstract}

\section{INFOBOX 1}

\section{Klinische Symptome der Minderperfusion}

- neurologisch: Verwirrtheit, Somnolenz, Agitation, Delir

- kardiovaskulär: Tachykardie (> 100 Schläge/min), Hypotension (systolischer Blutdruckwert $\leq 100 \mathrm{mmHg}$ ), Brustschmerzen, Dyspnoe

- pulmonal: Tachypnoe (Atemfrequenz $\geq 22 / \mathrm{min}$ )

- renal: Oligurie/Anurie

- Haut: marmorierte Extremitäten

- Blut: erhöhtes Serum-Laktat

Der Schock lässt sich in 4 Kategorien einteilen ( $\bullet$ Abb. 1):

- Der distributive Schock umfasst den septischen, den anaphylaktischen, den neurogenen und den endokrinen Schock. Hier kommt es durch den Verlust des Gefäßtonus und/oder durch eine erhöhte Gefäßpermeabilität zu einem Volumenverlust aus dem Gefäßsystem in das Gewebe; es besteht also ein relativer Volumenmangel.

- Der hypovolämische Schock lässt sich in einen hämorrhagischen (traumatisch/nicht traumatisch) und einen nicht hämorrhagischen Schock unterteilen.
- Der kardiogene Schock kann infarkt- oder nicht infarktbedingt sein (z. B. dekompensierte Klappenvitien, Arrhythmien, Perimyokarditis) und ist meist gekennzeichnet durch ein verringertes Herzzeitvolumen aufgrund eines Pumpversagens.

- Der obstruktive Schock führt entweder aufgrund einer kardialen (z. B. Perikardtamponade) oder einer extrakardialen Ursache (z. B. Spannungspneumothorax) zu einem verminderten kardialen Auswurf.

\section{Akutmedizinische Vorbereitung: Schockraum}

Wird vom Rettungsdienst ein Patient im Schock angekündigt, startet die Vorbereitung:

- die Schockraumversorgung wird aktiviert,

- je nach beschriebenem Befund (z. B. Polytrauma) versammelt sich ein Team aus den verschiedenen Fachdisziplinen [2],

- ein Team-Time-Out bzw. Briefing findet statt, um die Rollen der Teammitglieder abzustimmen, und

- ein Team-Leader wird benannt, der die Maßnahmen koordiniert.

\section{Merke}

Hausinterne standardisierte Vorgehensweisen (SOPs, „standard operation procedures") tragen insbesondere in der Akutsituation dazu bei, dass logistische und fachübergreifende Maßnahmen verfügbar und schnell abrufbar sind.

Aufgrund der raschen Dynamik des klinischen Zustandes und des zeitkritischen Managements sind diagnostische und therapeutische Maßnahmen im Schockraum bzw. im Herzkatheterlabor vorzubereiten (siehe auch Infobox 2). Wurde bereits präklinisch ein infarktbedingter kardiogener Schock erkannt, wird der Patient idealerweise in das Herzkatheterlabor mit integriertem Notfall- bzw. Intensivteam eingewiesen. Vorzubereiten sind bei jedem Patienten im Schock: 
Schock

distributiv
- Sepsis
- Anaphylaxie
- Neurogen
- Endokrin

hypovolämisch
- hämorrhagisch-
traumatisch
- hämorrhagisch-
atraumatisch
(z. B. gastrointes-
tinale Blutung)
- nicht hämor-
rhagisch

kardiogen
- infarktbedingt
- TakoTsubo-
Kardiomyopathie
- (Peri)Myokarditis
- dekompensiertes
Klappenvitium

obstruktiv
- Perikarderguss/
-tamponade
- Lungenembolie
- Spannungs-
pneumothorax

- Abb. 1 Ätiologische Einteilung der verschiedenen Schockformen [5].

- invasive und nicht invasive Beatmungsmöglichkeiten,

- Medikamente und Utensilien für Atemwegsmanagement und Gefäßzugang (v. a. Arterienkatheter und zentraler Venenkatheter),

- zwei Thoraxdrainagen (24 - 32 Charrière) bei V.a. Thoraxtrauma oder Pneumothorax [2],

- Beckengurt,

- Perikardpunktionsset,

- Tourniquet.

Die Versorgung eines (poly-)traumatisierten Patienten im hämorrhagischen Schock erfolgt im Schockteam. Bereitzustellen bzw. anzufordern sind hier:

- Blut- (Erythrozytenkonzentrate der Blutgruppe O) und Gerinnungsprodukte (z. B. Prothrombinkonzentrat, Tranexamsäure, Thrombozytenkonzentrate)

- Infusionswärmer mit der Möglichkeit zur Druckinfusion, um eine rasche Transfusion von erwärmten Konserven oder kristalloiden Flüssigkeiten zu ermöglichen

- kreislaufstabilisierende Medikamente (z. B. Noradrenalin) zur kontinuierlichen Gabe mittels Spritzenpumpe

\section{INFOBOX 2}

\section{Bildgebung}

Die sonografische bzw. echokardiografische Beurteilung sollte niemals isoliert, sondern stets im klinischen Kontext erfolgen.

- Sonografie

Initial sollte jeder Patient mit Thorax- und Abdominaltrauma mittels Ultraschall untersucht werden (eFAST, „extended focused assessment with sonography for trauma“). Wiederholungssonografien sind indiziert, wenn eine Computertomografie des Körperstamms nicht zeitnah durchführbar ist [2].
- Echokardiografie

Bei Patienten mit unklarer hämodynamischer Instabilität - insbesondere, wenn eine kardiale Ursache vermutet wird - ist eine transthorakale Echokardiografie zu veranlassen [3]. Bei einem infarktbedingten kardiogenen Schock soll umgehend nach Aufnahme eine fokussierte Echokardiografie erfolgen, ohne dass die Herzkatheteruntersuchung verzögert wird [4].

Kritisch kranke bzw. hämodynamisch instabile Patienten werden nach dem ABCDE-Schema (Airway-BreathingCirculation-Disability-Exposure) evaluiert, Traumapatienten nach dem C-ABCDE-Schema (Circulation-AirwayBreathing-Circulation-Disability-Exposure) ( Tab. 1).

\section{Merke Überbringung in die weiterversorgende Einheit. \\ Differenzialdiagnostisches Management}

Die klare Kommunikation der erhobenen Befunde (Call-Outs, Readback, Hearback) im Team ist unabdingbar. Ziele der Schockraumversorgung sind: schnellstmögliche Diagnostik, Stabilisierung des Patienten und

\section{Initiale Untersuchung}

Das führende Zeichen des Schocks ist in der Regel die Hypotension. Häufig finden sich in der initialen Untersuchung bereits Hinweise auf eine mögliche Ursache des Kreislaufschocks. Die (Fremd-) Anamnese sollte auf mögliche Auslöser fokussieren. So kann bei plötzlichem Brustschmerz und/oder Dyspnoe ein akutes Koronarsyndrom vorliegen. Für einen Schock nach Thoraxtrauma kommen mehrere Ätiologien in Frage: Hämatothorax, Spannungspneumothorax, Aortendissektion, Lungen- und Herzkontusion sowie Perikardtamponade. Auch die klini- 
- Tab. 1 (C)ABCDE-Algorithmus zur initialen Untersuchung und Stabilisierung.

\begin{tabular}{|l|l|l|}
\hline Algorithmus & klinische Untersuchung & Sofortmaßnahmen (Beispiele) \\
\hline C - „Circulation“ & äußere Blutung? & Kompression, Anlage Tourniquet \\
\hline A - Atemweg (+ HWS) & $\begin{array}{l}\text { verlegter/gefährdeter Atemweg? } \\
\text { HWS-Druckschmerz? }\end{array}$ & $\begin{array}{l}\text { Atemwegssicherung/Intubation, Anlage einer } \\
\text { Zervikalstütze/manuelle Immobilisation }\end{array}$ \\
\hline B - Belüftung & Auskultation (beidseits?), periphere O $_{2}$-Sättigung & Sauerstoffgabe, Beatmung, Anlage Thoraxdrainage \\
\hline C - „Circulation“ & Rekapillarisierungszeit, Blutdruckmessung, \\
\hline D - „Disability“/Defizit & Herzfrequenz, 12-Kanal-EKG & Anlage venöser und arterieller Zugänge, Druckinfusion/ \\
\hline E - Exposition & Hinweis auf fokales neurologisches Defizit & Transfusion, Anlage Beckengurt \\
\hline
\end{tabular}

sche Untersuchung kann relevante Hinweise liefern: z. B. ein einseitig aufgehobenes Atemgeräusch bei einem Spannungspneumothorax oder Rasselgeräusche bei einem kardiogenen Schock.

\section{Fokussierte und strukturierte Sonografie}

Sie soll als Erweiterung der körperlichen Untersuchung bei jedem Patienten im Schock durchgeführt werden [2 -4] und Bestandteil der Erstuntersuchung sein [6]; sie kann direkt im Anschluss an das initiale Assessment oder parallel dazu erfolgen. Ziel der Sonografie ist, schnellstmöglich einen gezielten (weiteren) diagnostischen und therapeutischen Pfad einzuleiten. Es bietet sich eine strukturierte Vorgehensweise an ( $\mathbf{A b b} .2)[5,6]$.

Vena cava inferior:

Initial wird die Vena cava inferior ( $\mathrm{VCl}$ ) anhand des Diameters und der Atemvariabilität beurteilt [3, 6].

- Eine kollaptische und schmale VCI (<10 mm) spricht für einen Volumenmangel und erfordert die unverzügliche Volumentherapie sowie das Auffinden der Ursache. Bei einem traumatisch bedingten hypovolämischen Schock ist mittels eFAST freie Flüssigkeit intraabdominell oder intrathorakal detektierbar.

- Zeigt sich dagegen eine erweiterte, atemstarre VCI (>22 mm) ist in den meisten Fällen (nicht in allen, wie z. B. bei schwerer Trikuspidalklappeninsuffizienz) ein hypovolämischer Schock sehr unwahrscheinlich [7]. Mit der fokussierten Echokardiografie lässt sich nachweisen, welcher Befund vorliegt:

- Perikardtamponade,

- eingeschränkte linksventrikuläre und/oder rechtsventrikuläre Pumpfunktion bzw. Rechtsherzbelastungszeichen,

- Dissektion der Aorta ascendens,

- höhergradige Klappenvitien (v. a. Aortenklappenstenose, Mitralklappeninsuffizienz).

\section{Merke}

Vorteile der bettseitigen Sonografie sind: rasche Differenzierung, gezielter Therapiebeginn sowie die Möglichkeit der Re-Evaluation ohne Belastung oder Gefährdung des instabilen Schockpatienten.

\section{Erweiterte Diagnostik beim Kreislaufschock}

\section{2-Kanal-EKG:}

Dies gehört zur erweiterten Diagnostik (fast) immer dazu. Bei klinischen Zeichen eines akuten Koronarsyndroms bzw. eines infarktbedingten kardiogenen Schocks muss es spätestens innerhalb von 10 Minuten nach Krankenhausaufnahme geschrieben sein [4].

Laborchemische Untersuchungen:

Sie umfassen Blutbild (Differenzialblutbild), Entzündungsparameter (inklusive Procalcitonin), Cholestaseparameter, Transaminasen, Nierenretentions- und Gerinnungsparameter sowie venöse (ggf. arterielle) Blutgasanalyse. Blutgruppenbestimmung und Abnahme von Kreuzblut sind je nach Ätiologie ebenfalls wichtig. Spezielle kardiale Marker (v. a. Troponin und/oder NT-proBNP) sind abhängig von der vermuteten Ursache zu bestimmen. Bei Verdacht auf einen septischen Schock müssen Blutkulturen (mindestens 2 Paar) sowie eine Urinprobe abgenommen werden [8].

\section{Merke}

Eine weitere Diagnostik (z. B. Computertomografie, Herzkatheteruntersuchung) sollte abhängig von der Arbeitsdiagnose und der hämodynamischen Ist-Situation erfolgen. 


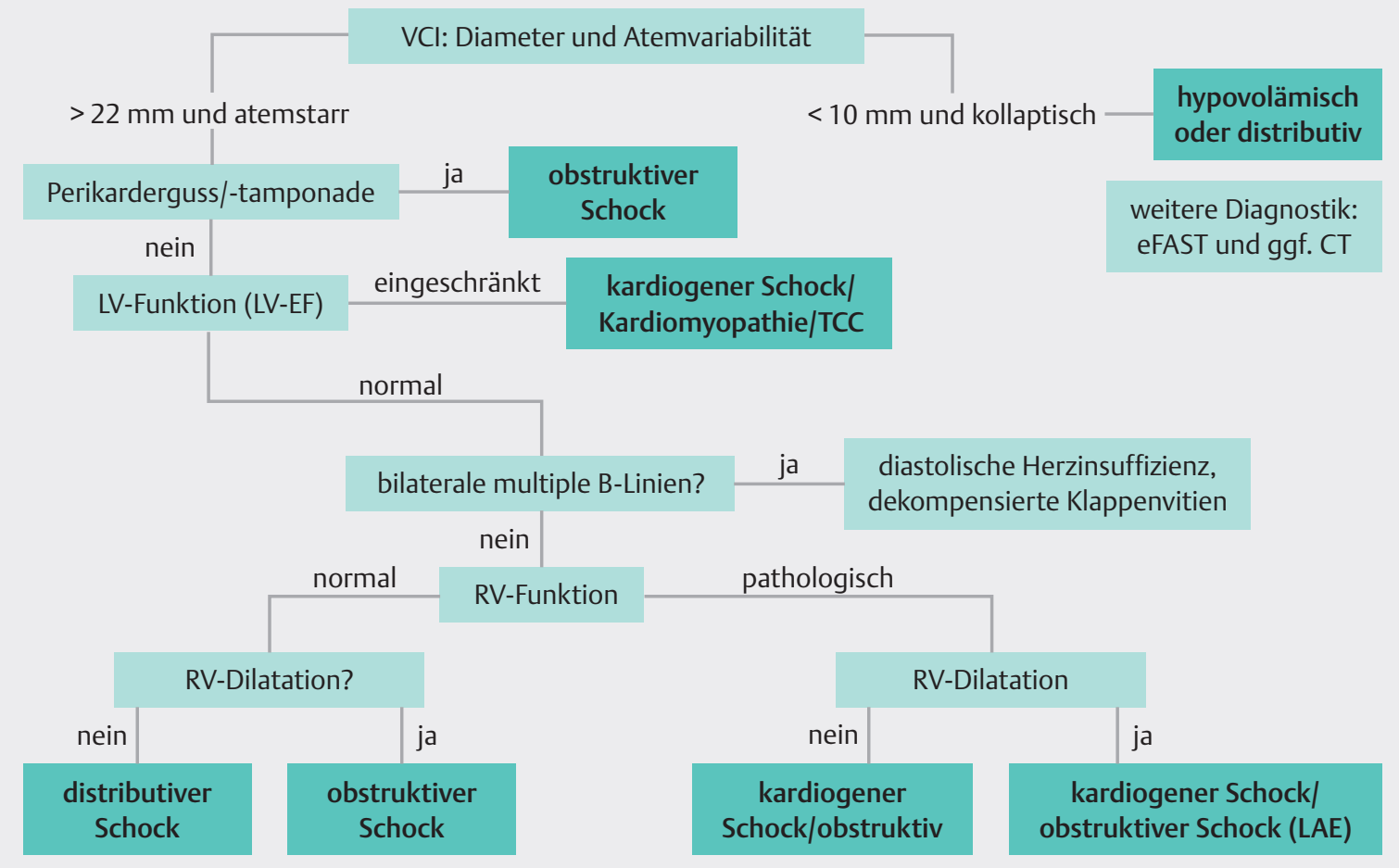

- Abb. 2 Fokussierte Sonografie im Rahmen des Schockgeschehens [5]. eFAST: extended focused assessment with sonography for trauma, LAE: Lungenarterienembolie, LVEF: linksventrikuläre Ejektionsfraktion, RV: rechter Ventrikel, TCC: Takotsubo-Kardiomyopathie, $\mathrm{VCl}$ : Vena cava inferior.

\section{Differenziertes Management der verschiedenen Schockformen}

\section{Distributiver Schock}

\section{Septischer Schock}

Die Sepsis ist definiert als lebensbedrohliche Organdysfunktion aufgrund einer Fehlregulation der Wirtantwort auf eine Infektion [8]. Der septische Schock geht einher mit einer zirkulatorischen und zellulären bzw. metabolischen Dysfunktion sowie deutlich erhöhter Mortalität. Er erfüllt beide Kriterien:

- Notwendigkeit der Vasopressortherapie, um bei persistierender Hypotonie einen mittleren arteriellen Druck (MAP) $\geq 65 \mathrm{mmHg}$ aufrechtzuerhalten, sowie

- erhöhter Serum-Laktatspiegel $\geq 2 \mathrm{mmol} / \mathrm{l}$ trotz adäquater Volumensubstitution.

Die Initialtherapie zeigt die Infobox 3. Der Schweregrad der Organdysfunktion ist mittels SOFA-Score („sequential organ failure assessment") bestimmbar. Da dieser recht komplex ist, wird für Notaufnahme, Normalstation und Präklinik der vereinfachte „quick“-SOFA (qSOFA)-Score empfohlen [9, 10]. Er hat eine relativ hohe Spezifität bei jedoch geringerer Sensitivität. Der qSOFA-Score umfasst:

- Bewusstsein (Glasgow Coma Scale <15),

- Atemfrequenz ( $\geq 22 / \mathrm{min})$ und

- systolischen Blutdruck ( $\leq 100 \mathrm{mmHg})$.

Ein qSOFA-Score von $\geq 2$ deutet auf eine deutlich erhöhte Mortalität hin.

Cave

Ein negativer qSOFA-Score darf nicht dazu führen, dass eine Sepsis verzögert behandelt wird. Trozt adäquater Therapie beträgt die KH-Sterblichkeit des septischen Schocks weiterhin zw. $40-60 \%[8,9]$.

\section{INFOBOX 3}

Die initiale Therapie („Sepsis-Bundles“) umfasst:

- Volumentherapie mit kristalloider Flüssigkeit, mindestens $30 \mathrm{ml} / \mathrm{kgKG}$ innerhalb von 3 Stunden bei Hypotension oder erhöhtem Laktat ( $\geq 2 \mathrm{mmol} / \mathrm{l}$ ) [8]. Das Monitoring der Flüssigkeitstherapie bzw. der Volumenreagibilität stellt für 
viele Intensivmediziner bis heute weiterhin eine Herausforderung dar [3, 7].

- Beginn einer Antibiotikatherapie innerhalb der ersten Stunde nach Abnahme von zwei Blutkultur-Sets (aerob und anaerob), zunächst mittels Breitspektrumantibiotikum; tägliche Reevaluation und sobald wie möglich Deeskalation der Antibiotikatherapie.

- Gabe von Noradrenalin (ggf. in Kombination mit Vasopressin), wenn trotz adäquater Flüssigkeitsgabe kein MAP von $\geq 65 \mathrm{mmHg}$ erzielt wurde.

Additiv zu Noradrenalin kann Vasopressin oder ggf. Adrenalin zum Einsatz kommen. Vasopressin oder Vasopressin-Analoga (z. B. Terlipressin) werden v. a. gegeben, um Noradrenalin einzusparen und dessen potenzielle Toxizität zu reduzieren [8]. Sollte es trotz adäquater Katecholamintherapie nicht zu einer hämodynamischen Stabilisierung kommen, wird eine adjunktive Therapie mit Steroiden empfohlen. Die Frage nach dem Nutzen der Co-Therapie mit Steroiden im Rahmen der Sepsistherapie (Hydrocortison \pm Fludrocortison) ist weiterhin Gegenstand aktueller Diskussionen. In ausgewählten Einzelfällen kann man in erfahrenen Zentren unter individueller Nutzen-Schaden-Analyse eine ECMO (extrakorporale Membranoxygenierungs)-Therapie erwägen.

\section{Anaphylaktischer Schock}

Die (Fremd-)Anamnese und die klinische Untersuchung geben hier sehr häufig wichtige Hinweise auf die Genese bzw. auf eine Exposition gegenüber einem Auslöser. Ein anaphylaktischer Schock kann vermutet werden bei

- plötzlichem Beginn und raschem Fortschreiten der Symptome,

- einem Atemwegs-/Belüftungs- oder „Circulation“Problem,

- einer Haut-/Schleimhautreaktion (v. a. Urtikaria und Angioödem) [11].

\section{Cave}

Bis zu $20 \%$ der Patienten mit anaphylaktischem Schock zeigen keine Hautreaktionen!

Häufigste Auslöser für den anaphylaktischen Schock im Kindesalter sind Nahrungsmittel und im Erwachsenenalter Insektengifte. Pathophysiologisch findet bei der Anaphylaxie eine IgE-vermittelte Reaktion (Typ I) statt. Durch Anbinden des Allergens an spezifische auf Mastzellen oder Granulozyten gebundene IgE-Antikörper kommt es zur Degranulation und Mediatorenfreisetzung. Histamin, Leukotriene, Prostaglandine und weitere Mediatoren bewirken, dass sich die Gefäßpermeabilität erhöht und der Gefäßtonus sinkt, was letztendlich zum distributiven Schock führt.
Die S2k-Leitlinie zu Akuttherapie (siehe auch Infobox 4) und Management der Anaphylaxie benennt vier Schweregrade [11]. Ab Grad II kommt es zu Symptomen im Bereich des Herz-Kreislauf-Systems mit Hypotension und Tachykardie, im Stadium III liegt ein Schock vor, im Stadium IV ein Kreislaufstillstand. Symptome im Bereich der Haut, des Gastrointestinal- und des Respirationstraktes können (müssen aber nicht) einer Kreislaufdepression vorausgehen.

\section{INFOBOX 4}

Das wichtigste Medikament in der Behandlung des anaphylaktischen Schocks ist Adrenalin.

Die Dosierung bei einem nicht reanimationspflichtigen Patienten beträgt 0,3-0,5 mg i. m. ab einem Körpergewicht von $30 \mathrm{~kg}$. Die primär intramuskuläre Applikation wird empfohlen, weil so das Risiko schwerer kardiovaskulärer Nebenwirkungen erheblich geringer ist. Nur wenn nach 2-3-maliger Gabe (Repetition alle 5-10 min) keine Besserung eintritt bzw. eine respiratorische und/oder hämodyamische Dekompensation droht, sollte Adrenalin intravenös appliziert werden (titrierte Boligaben $10-100 \mu \mathrm{g}$ oder Dauerinfusion 0,05-1 $\mu \mathrm{g} / \mathrm{kgKG} / \mathrm{min}$ ) [11, 12]. Zusätzlich ist die Gabe kristalloider Infusionslösungen (bis zu 2 - 3 Liter) über großkalibrige intravenöse Zugänge sinnvoll [11].

Anti-Histaminika (wie $\mathrm{H}_{1}$ - bzw. $\mathrm{H}_{2}$-Rezeptorblocker) zielen lediglich ab auf die Haut- und Schleimhautsymptome. Kortikosteroide wirken nur verzögert und spielen deshalb in der Akutbehandlung eine untergeordnete Rolle. Aufgrund der Gefahr einer verzögerten Reaktion bzw. einer Rückkehr der Symptome sollte dennoch die Gabe von Kortison erfolgen: Prednisolon initial 250-1000 mg i. v., danach $1 \mathrm{mg} / \mathrm{kgKG}$ per os für 2 Tage [11].

\section{Neurogener Schock}

Der neurogene Schock ist gekennzeichnet durch Hypotension und Bradykardie [13]. Es kommt zum Ausfall der sympathischen Efferenzen bei erhaltenem Parasympathikus. Die häufigste Ursache des neurogenen Schockes ist eine spinale Verletzung oberhalb des 6 . Thorakalsegments (Th6) bei Verletzungen der Brustwirbelsäule. Aber auch zentrale Pathologien wie Schädigung des Kreislaufzentrums im Bereich des Hirnstamms durch Einblutung, Ischämie oder Einklemmung führen zum neurogenen Schock.

\footnotetext{
Der neurogene Schock darf nicht mit dem spinalen Schock verwechselt werden! Der spinale Schock bezeichnet die Kombination aus Reflexausfall, Tonusverlust und Sensibilitätsverlust unterhalb der Stelle einer spinalen Läsion.
} 
Diagnostische Hinweise auf einen neurogenen Schock sind Hypotonie, Bradykardie, eine warme Haut sowie meist ein entsprechendes Trauma. Die Behandlung des Auslösers hat erste Priorität. Die Hypotonie wird durch Volumengabe und Vasopressoren therapiert.

\section{Endokriner Schock}

Im Rahmen einer Addison-Krise kann ein distributiver Schock auftreten. Wegweisende Symptome sind Übelkeit, Erbrechen, Hyponatriämie und Hyperkaliämie. Die häufigsten Auslöser einer Addison-Krise sind Infektionen (insbesondere Gastroenteritis), Operationen, Schmerzen oder emotionaler Stress. Die Therapie besteht aus Volumen- und Elektrolytsubstitution sowie der Gabe von Hydrocortison (initial $100 \mathrm{mg}$ i. v., danach $200 \mathrm{mg}$ täglich i. v.). Ggf. ist auch die Gabe von Glukose notwendig [14].

\section{Hypovolämischer Schock}

\section{Hämorrhagischer Schock, traumatisch}

Die häufigste Todesursache bei Polytrauma-Patienten ist der hämorrhagische Schock. Äußere Blutungen sollten möglichst gestoppt werden (C-ABCDE). Ist dies durch direkte Kompression nicht erreichbar, ist ein Tourniquet anzulegen. Im Gegensatz zum septischen Schock wird im hämorrhagischen Schock (sofern kein Schädel-HirnTrauma vorliegt) ein systolischer Blutdruck von $90 \mathrm{mmHg}$ angestrebt (permissive Hypotonie), bis die Blutung gestoppt ist. Neben der Gabe von Kristalloiden wird eine Vasopressortherapie (Noradrenalin) empfohlen [2].

\section{Merke}

Insbesondere der Wärmeerhalt hat bei Patienten im hämorrhagischen Schock eine große Bedeutung, da eine Hypothermie die Gerinnungssituation weiter verschlechtert.

Eine Rescue- bzw. Ultima-Ratio-Maßnahme wird aktuell nur in ausgewählten Zentren durchgeführt, nämlich die temporäre endovaskuläre Ballonokklusion der Aorta (REBOA: „resuscitative endovascular balloon occlusion of the aorta“) [2]. Hierbei wird die Aorta durch einen Ballon komplett oder teilweise verschlossen. Diese Methode kann als überbrückendes Verfahren („bridge to surgery“) zum Einsatz kommen, wenn die Blutungsquelle unterhalb des Diaphragmas (Zone II und III der Aorta) liegt und nicht anders zu kontrollieren ist [15].

\section{Hämorrhagischer Schock, nicht traumatisch}

Ein hämorrhagischer Schock ohne Trauma kann bei den verschiedensten Formen der „inneren Blutung“ auftreten. Die häufigsten Blutungsquellen finden sich im oberen Gastrointestinaltrakt. Hier wird zwischen einer nicht varikösen (z. B. Magenulkus) und varikösen Blutung (meist Patienten mit bekannter Leberzirrhose; siehe auch Infobox 5) unterschieden. Bei Patienten im hämor- rhagischen Schock durch eine Blutung aus dem oberen Gl-Trakt spielt neben der Kreislaufstabilisierung auch die initiale Einschätzung des Atemwegs eine große Rolle; bei schwerer Blutung ist eventuell eine endotracheale Intubation vor der Notfallendoskopie notwendig, um das Aspirationsrisiko zu vermindern. Bezüglich des Gerinnungsmanagements nehmen Patienten mit einer vorbestehenden Leberzirrhose eine Sonderstellung ein [16].

\section{INFOBOX 5}

Bei Patienten mit vermuteter akuter Ösophagusvarizenblutung ist an Folgendes zu denken:

- frühzeitige Antibiotikaprophylaxe (z. B. Ceftriaxon $2 \mathrm{~g}$ i. v. zur Verhinderung der bakteriellen Translokation),

- Vasokonstriktor (z. B. 1 - 2 mg Terlipressin als i. v. Bolus, dann $1 \mathrm{mg}$ alle 6 Stunden für 3 - 5 Tage),

- Transfusionsziel (Hämoglobin-Wert 7 - $9 \mathrm{~g} / \mathrm{dl}$, restriktive Transfusionsstrategie),

- Notfallendoskopie (vorher 250 mg Erythromycin i. v. einmalig) mit endoskopischer Blutstillung,

- TIPS-Anlage (transjugulärer intrahepatischer portosystemischer Shunt) bei refraktären/rezidivierenden Varizenblutungen.

\section{Hypovolämischer Schock, nicht hämorrhagisch}

Der Verlust großer Flüssigkeitsmengen im Rahmen schwerer Gastroenteritiden, einer Hyperthermie oder bei Verbrennungen kann ebenfalls zum hypovolämischen Schock führen.

\section{Kardiogener Schock}

Der kardiogene Schock ist gekennzeichnet durch ein vermindertes Herzzeitvolumen. Die mit Abstand häufigste Ursache ist ein akuter Myokardinfarkt. Die deutsch-österreichische S3-Leitlinie „Infarktbedingter kardiogener Schock - Diagnose, Monitoring und Therapie“ wurde aktuell überarbeitet und ist derzeit eingereicht, die Revision jedoch noch nicht abgeschlossen [4]. Die folgenden Empfehlungen und Kommentare stammen aus der überarbeiteten Leitlinie.

\footnotetext{
Merke

Die wichtigste therapeutische Maßnahme ist die Revaskularisation mittels perkutaner Koronar-Intervention (PCI). Ein Zeitintervall von 90 Minuten zwischen Diagnosestellung und $\mathrm{PCl}$ sollte nicht überschritten werden.
}

Bei koronarer Mehrgefäßerkrankung und mehreren relevanten Stenosen (> $70 \%$ ) soll im Rahmen der PCI nur die infarktverursachende Läsion („culprit lesion“) interve- 
niert werden [17]. Die folgenden therapeutischen Maßnahmen sind bei kardiogenem Schock zu beachten:

- Bei Diagnosestellung werden Acetylsalicylsäure 250 $500 \mathrm{mg}$ i. v. und ein Antikoagulans (meist unfraktioniertes Heparin i. v.) gegeben.

- Ein ADP-Rezeptorantagonist soll bei PCI mit Stenting verabreicht werden.

- Als Inotropikum der Wahl wird Dobutamin, als Vasopressor Noradrenalin empfohlen.

- Im Katecholamin-refraktären Schockzustand sollte initial Levosimendan gegenüber PhosphodiesteraseIII-Inhibitoren bevorzugt werden.

Die intraaortale Ballongegenpulsation (IABP) als mechanisches Herz-Kreislauf-Unterstützungssystem wird bei infarktbedingtem kardiogenem Schock infolge eines alleinigen Pumpversagens nicht empfohlen. Sie kann jedoch bei Auftreten mechanischer Infarktkomplikationen (z. B. Ventrikelseptumdefekt) zur Verbesserung der Hämodynamik erwogen werden. Bei ausgewählten Patienten mit Pumpversagen, die sich durch eine medikamentöse HerzKreislauf-Unterstützung nicht stabilisieren lassen, kann ein temporäres mechanisches Unterstützungssystem (z. B. linksventrikuläre Mikroaxialpumpe oder Extracorporeal Life Support System) implantiert werden.

Selten ist eine Myokarditis Ursache des kardiogenen Schocks. Patienten mit Verdacht auf Myokarditis und hämodynamische Instabilität sollten an Zentren mit entsprechender Expertise behandelt werden. Neben der medikamentösen Herzinsuffizienz-Therapie ist ggf. die Implantation eines Herz-Kreislauf-Unterstützungssystems notwendig [18].

\section{Obstruktiver Schock}

Die drei häufigsten Ursachen für einen obstruktiven Schock sind Perikardtamponade, Lungenarterienembolie und Spannungspneumothorax.

- Perikardtamponade

Hier muss durch sofortige Entlastung mittels Perikardpunktion oder ggf. Perikardiotomie therapiert werden.

- Lungenarterienembolie

Diese kann bei deutlichen echokardiografischen Zeichen der Rechtsherzbelastung vermutet werden. Bei Patienten im Schock wird die fokussierte Echokardiografie als initiale Diagnostik empfohlen [19]. Ist eine CT-Angiografie nicht direkt verfügbar oder ist der Patient instabil, sollte die Therapie sofort nach der Echokardiografie eingeleitet werden (systemische Thrombolysetherapie, ggf. kathetergestützte Lysetherapie oder chirurgische Embolektomie).

\section{- Spannungspneumothorax}

Dieser muss durch umgehende Entlastung behandelt werden. Neben der klinischen Untersuchung ist die Thoraxsonografie differenzialdiagnostisch wegweisend: hier sind auf der betroffenen Seite kein Lungengleiten und kein Lungenpuls nachweisbar [5, 6].

\section{KERNAUSSAGEN}

- Der Schock ist gekennzeichnet durch ein Missverhältnis zwischen Sauerstoffangebot und Sauerstoffbedarf.

- Pathophysiologisch können vier Gruppen unterschieden werden: distributiv, hypovolämisch, kardiogen und obstruktiv.

- Das initiale Assessment erfolgt nach dem (C)ABCDE-Schema. Parallel oder in direktem Anschluss sollte die fokussierte Sonografie bzw. Echokardiografie durchgeführt werden.

- Eine spezifische Therapie setzt die ätiologische Abklärung des Schockgeschehens voraus.

- Der Patient im Schock muss schnellstmöglich im Schockraum diagnostiziert und stabilisiert werden. Das innerklinische Schockraumteam muss daher den Schockraum entsprechend vorbereiten. Ein Briefing über die Aufgabenverteilung erleichtert die Kommunikation in der Akutsituation.

\section{Interessenkonflikt}

Dr. Hempel gibt an, dass kein Interessenkonflikt besteht. Prof. Dr. Michels hat Honorare für Vorträge von folgenden Firmen erhalten: Pfizer, Novartis, Servier, Zoll, Getinge und Orion Pharma. Er gibt an, dass hierdurch kein Konflikt mit der vorliegenden Arbeit besteht.

\section{Autorinnen/Autoren}

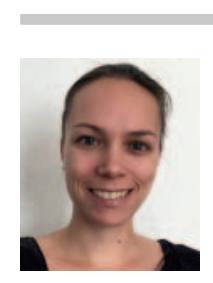

\section{Dr. med. Dorothea Hempel}

Leitende Oberärztin der Zentralen Notaufnahme mit Aufnahmestation der Universitätsklinik Magdeburg.

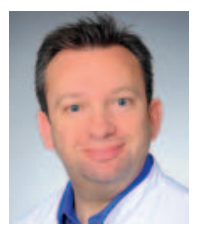

\section{Prof. Dr. med. Guido Michels}

Oberarzt der Klinik III für Innere Medizin und Leiter der kardiologischen Intensivstation im Herzzentrum der Medizinischen Fakultät und Uniklinik Köln. Mitglied des Leitlinienkomitees der deutsch-österreichischen S3-Leitlinie „Infarktbedingter kardiogener Schock - Diagnose, Monitoring und Therapie“.

\section{Korrespondenzadresse}

\section{Prof. Dr. med. Guido Michels}

Klinik III für Innere Medizin der Universität zu Köln

Kerpener Str. 62

50937 Köln

guido.michels@uk-koeln.de 
Literatur

[1] Vincent JL, De Backer D. Circulatory shock. N Engl J Med 2013; 369: $1726-1734$

[2] Polytrauma Guideline Update Group. Level 3 guideline on the treatment of patients with severe/multiple injuries: AWMF Register-Nr. 012/019. Eur J Trauma Emerg Surg 2018; 44 (1): $3-271$

[3] Marx G. New AWMF S3-guidelines for volume therapy: goodbye emotions - welcome evidence. Med Klin Intensivmed Notfmed 2015; 110: 108-109

[4] Werdan K, Russ M, Buerke M et al. Cardiogenic shock due to myocardial infarction: diagnosis, monitoring and treatment: a German-Austrian S3 Guideline. Dtsch Arztebl Int 2012; 109: $343-351$

[5] Hempel D, Pfister R, Michels G. Hemodynamic monitoring in intensive care and emergency medicine: Integration of clinical signs and ultrasound findings. Med Klin Intensivmed Notfmed 2016; 111: $596-604$

[6] Hempel D, Pfister R, Michels G. Structured bedside-ultrasound in intensive care medicine. Med Klin Intensivmed Notfmed 2017; 112: 741 - 758

[7] Adler C, Treskatsch S, Pfister R et al. Estimation of Preload Dependence in Intensive Care - Step by Step. Dtsch Med Wochenschr 2019; 144: $340-345$

[8] Rhodes A, Evans LE, Alhazzani W et al. Surviving Sepsis Campaign: International Guidelines for Management of Sepsis and Septic Shock: 2016. Intensive Care Med 2017; 43: 304- 377

[9] Freund Y, Lemachatti N, Krastinova E et al. Prognostic Accuracy of Sepsis-3 Criteria for In-Hospital Mortality Among Patients With Suspected Infection Presenting to the Emergency Department. JAMA 2017; 317: 301 - 308

[10] Singer M, Deutschman CS, Seymour CW et al. The Third International Consensus Definitions for Sepsis and Septic Shock (Sepsis-3). JAMA 2016; 315: $801-810$
[11] Ring J, Beyer K, Biedermann T et al. Guideline for acute therapy und management of anaphylaxis. S2 guideline of DGAKI, AeDA, GPA, DAAU, BVKJ, ÖGAI, SGAI, DGAI, DGP, DGPM, AGATE and DAAB. Allergo J Int 2014; 23: 96-112

[12] Wood JP, Traub S], Lipinski C. Safety of epinephrine for anaphylaxis in the emergency setting. World J Emerg Med 2013; 4: $245-251$

[13] Popa C, Popa F, Grigorean VT et al. Vascular dysfunctions following spinal cord injury. J Med Life 2010; 3: 275- 285

[14] Arlt W, Society for Endocrinology Clinical C. Society for Endocrinology Endocrine Emergency Guidance: Emergency management of acute adrenal insufficiency (adrenal crisis) in adult patients. Endocr Connect 2016; 5: G1 - G3

[15] Brenner M, Teeter W, Hoehn M et al. Use of Resuscitative Endovascular Balloon Occlusion of the Aorta for Proximal Aortic Control in Patients With Severe Hemorrhage and Arrest. JAMA Surg 2018; 153: $130-135$

[16] Götz M, Anders M, Biecker E et al. S2k Guideline Gastrointestinal Bleeding - Guideline of the German Society of Gastroenterology DGVS. Z Gastroenterol 2017; 55: 883-936

[17] Thiele H, Desch S. CULPRIT-SHOCK (Culprit Lesion Only PCI Versus Multivessel Percutaneous Coronary Intervention in Cardiogenic Shock): Implications on Guideline Recommendations. Circulation 2018; 137: 1314-1316

[18] Hajjar LA, Teboul JL. Mechanical Circulatory Support Devices for Cardiogenic Shock: State of the Art. Crit Care 2019; 23: 76

[19] Konstantinides SV, Torbicki A, Agnelli G et al. 2014 ESC guidelines on the diagnosis and management of acute pulmonary embolism. Eur Heart J 2014; 35: 3033 - 3069, 3069a - 3069k

\section{Bibliografie}

DOI https://doi.org/10.1055/a-0828-9675

Dtsch Med Wochenschr 2019; 144: 884-891

(c) Georg Thieme Verlag KG, Stuttgart · New York ISSN 0012-0472 